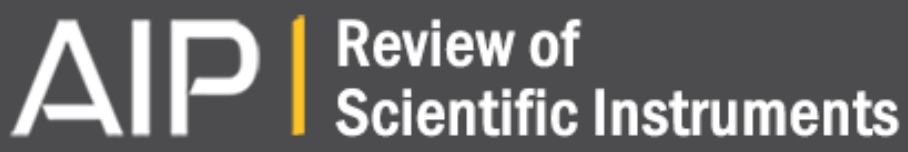

\section{An upgrade of the magnetic diagnostic system of the DIII-D tokamak for non- axisymmetric measurements}

J. D. King, E. J. Strait, R. L. Boivin, D. Taussig, M. G. Watkins, J. M. Hanson, N. C. Logan, C. Paz-Soldan, D. C. Pace, D. Shiraki, M. J. Lanctot, R. J. La Haye, L. L. Lao, D. J. Battaglia, A. C. Sontag, S. R. Haskey, and J. G. Bak

Citation: Review of Scientific Instruments 85, 083503 (2014); doi: 10.1063/1.4891817

View online: http://dx.doi.org/10.1063/1.4891817

View Table of Contents: http://scitation.aip.org/content/aip/journal/rsi/85/8?ver=pdfcov

Published by the AIP Publishing

\section{Articles you may be interested in}

Radial profile measurements of plasma pressure-like fluctuations with the heavy ion beam diagnostic on the tokamak ISTTOKa)

Rev. Sci. Instrum. 85, 11D848 (2014); 10.1063/1.4891045

Development of a cross-polarization scattering system for the measurement of internal magnetic fluctuations in the DIII-D tokamaka)

Rev. Sci. Instrum. 85, 11D838 (2014); 10.1063/1.4887276

Measurements of the deuterium ion toroidal rotation in the DIII-D tokamak and comparison to neoclassical theorya)

Phys. Plasmas 19, 056107 (2012); 10.1063/1.3694656

Magnetohydrodynamic interference with the edge pedestal motional Stark effect diagnostic on DIII-D Rev. Sci. Instrum. 82, 033515 (2011); 10.1063/1.3568827

High performance advanced tokamak regimes in DIII-D for next-step experiments

Phys. Plasmas 11, 2616 (2004); 10.1063/1.1692133

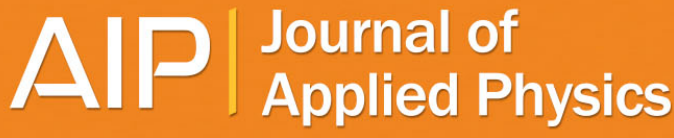

Journal of Applied Physics is pleased to announce André Anders as its new Editor-in-Chief 


\title{
An upgrade of the magnetic diagnostic system of the DIII-D tokamak for non-axisymmetric measurements
}

\author{
J. D. King, ${ }^{1}$ E. J. Strait, ${ }^{2}$ R. L. Boivin, ${ }^{2}$ D. Taussig, ${ }^{2}$ M. G. Watkins, ${ }^{2}$ J. M. Hanson, ${ }^{3}$ \\ N. C. Logan, ${ }^{4}$ C. Paz-Soldan, ${ }^{1}$ D. C. Pace, ${ }^{2}$ D. Shiraki, ${ }^{3}$ M. J. Lanctot ${ }^{2}$ R. J. La Haye ${ }^{2}$ \\ L. L. Lao, ${ }^{2}$ D. J. Battaglia, ${ }^{4}$ A. C. Sontag, ${ }^{5}$ S. R. Haskey, ${ }^{6}$ and J. G. Bak ${ }^{7}$ \\ ${ }^{1}$ Oak Ridge Institute for Science and Education, Oak Ridge, Tennessee 378300-8050, USA \\ ${ }^{2}$ General Atomics, P.O. Box 85608, San Diego, California 92186-5608, USA \\ ${ }^{3}$ Department of Applied Physics and Applied Mathematics, Columbia University, 116th and Broadway, \\ New York, New York 10027, USA \\ ${ }^{4}$ Princeton Plasma Physics Laboratory, P.O. Box 451, Princeton, New Jersey 08543-0451, USA \\ ${ }^{5}$ Oak Ridge National Laboratory, P.O. Box 2008, Oak Ridge, Tennessee 37831, USA \\ ${ }^{6}$ Plasma Research Laboratory, Research School of Physical Sciences and Engineering, \\ The Australia National University, Canberra, ACT 0200, Australia \\ ${ }^{7}$ Research and Development Division, National Fusion Research Center, Daejeon, South Korea
}

(Received 7 March 2014; accepted 21 July 2014; published online 7 August 2014)

\begin{abstract}
The DIII-D tokamak magnetic diagnostic system [E. J. Strait, Rev. Sci. Instrum. 77, 023502 (2006)] has been upgraded to significantly expand the measurement of the plasma response to intrinsic and applied non-axisymmetric "3D" fields. The placement and design of 101 additional sensors allow resolution of toroidal mode numbers $1 \leq \mathrm{n} \leq 3$, and poloidal wavelengths smaller than MARS-F, IPEC, and VMEC magnetohydrodynamic model predictions. Small 3D perturbations, relative to the equilibrium field $\left(10^{-5}<\delta B / B_{0}<10^{-4}\right)$, require sub-millimeter fabrication and installation tolerances. This high precision is achieved using electrical discharge machined components, and alignment techniques employing rotary laser levels and a coordinate measurement machine. A 16-bit data acquisition system is used in conjunction with analog signal-processing to recover non-axisymmetric perturbations. Co-located radial and poloidal field measurements allow up to $14.2 \mathrm{~cm}$ spatial resolution of poloidal structures (plasma poloidal circumference is $\sim 500 \mathrm{~cm}$ ). The function of the new system is verified by comparing the rotating tearing mode structure, measured by $14 \mathrm{~B}_{\mathrm{P}}$ fluctuation sensors, with that measured by the upgraded $B_{R}$ saddle loop sensors after the mode locks to the vessel wall. The result is a nearly identical $2 / 1$ helical eigenstructure in both cases. ( 2014 AIP Publishing LLC. [http://dx.doi.org/10.1063/1.4891817]
\end{abstract}

\section{INTRODUCTION}

Inductive loop and coil magnetic field measurement is the bedrock diagnostic for studying magnetically confined plasmas. These sensors provide a simple, robust measurement of static and fluctuating magnetic properties spanning the full range of operational space. The foundation of magnetic measurements dates back to the 19th century with the discovery of electromagnetic induction by Michael Faraday and, while not discussed in this paper, further application to laboratory plasma measurements has been thoroughly reviewed in previous works. ${ }^{1,2}$ Here we have taken the step to explore subtle changes to the tokamak magnetic field topology associated with small distortions in toroidal symmetry. A large disparity between Tesla equilibrium field and sub-Guass asymmetries $\left(10^{-5}<\delta B / B_{0}<10^{-4}\right)$ exists in tokamaks, requiring great care in sensor positioning, alignment and electronic instrumentation.

The tokamak is nominally an axisymmetric device and the full suite of axisymmetric magnetic field measurements on DIII-D is detailed in Ref. 20. Recently, small nonaxisymmetric, or "3D," fields have been found to be beneficial in fusion energy relevant plasma studies. Some examples of these results include resonant magnetic perturbations
(RMP) causing edge localized mode (ELM) suppression, ${ }^{3}$ non-resonant magnetic field (NRMF) induced torque increasing toroidal plasma rotation, ${ }^{4,5}$ and active feedback control of resistive wall modes (RWM). ${ }^{6,7}$

This article will discuss a large diagnostic upgrade that extends the non-axisymmetric magnetic field measurement capability of DIII-D. The physics objectives of this upgrade are diverse. The primary goal is measurement of the plasma response $^{8,9}$ to applied 3D fields. This measurement may prove critical to understanding how $\mathrm{n}=3$ RMPs suppress ELMs. Furthermore, the validation of 3D perturbed or equilibrium model predictions will be possible, allowing for accurate extrapolation to ITER. A second objective is a more complete measurement of the toroidal and poloidal structure of nonrotating magnetohydrodynamic (MHD) modes such as locked tearing modes ${ }^{10}$ and resistive wall modes. Furthermore, local electromagnetic torque between the plasma and applied fields may be directly measured, ${ }^{11}$ which can further elucidate error field braking effects and enable the optimization of necessary corrections. The eventual full reconstruction of non-axisymmetric equilibria will be possible. This upgrade is intended to improve axisymmetric equilibrium EFIT ${ }^{12}$ reconstructions, as well. Finally, a small subset of 6 high frequency response sensors was added to improve the detection of ELM 


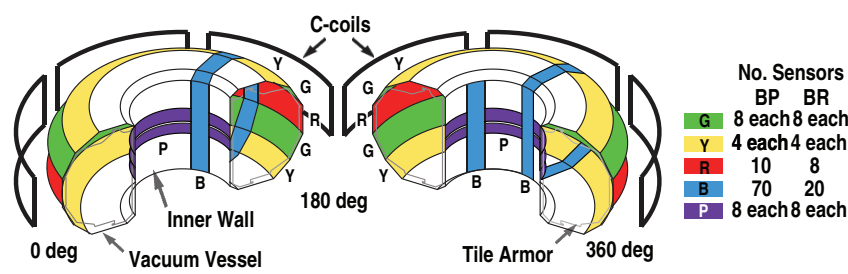

FIG. 1. Number and location of 3D sensors for each region of the DIII-D vacuum vessel.

precursors and small wavelength toroidal Alfven eigenmodes (TAE). ${ }^{13}$ Future application of these new capabilities to real-time feedback control may enable tokamak operation in previously inaccessible regimes by stabilizing RWM growth. ${ }^{14-16}$

Magnetic measurement of non-rotating, nonaxisymmetric plasma modes requires an additional dimension of sensor coverage. For rotating modes, if the phenomenon under study evolves much slower than the toroidal plasma rotation period, then all phases of the mode will pass a single location, allowing diagnosis of the structure from a single poloidal array at one toroidal angle. However, for static variations, all phases of the mode must be simultaneously measured toroidally to capture the full structure at a single time.

Figure 1 shows the number and location of sensors now used for 3D field measurements on DIII-D. Previously only low field side (LFS) simultaneous measurements of toroidal asymmetries with $\mathrm{n} \leq 2$ were resolvable in the bands labeled $\mathrm{G}$, and $\mathrm{n} \leq 3$ was only possible using a single midplane array in band $\mathrm{R}$. This meant only the toroidal component of the toroidal plasma response could be resolved at the LFS midplane for $\mathrm{n}=3$ RMP ELM suppression experiments. To study detailed screening-kink interactions, the full 3D plasma response must be measured. This motivated extending $n=3$ coverage to all but the upper and lower divertor regions. Using synchronous detection, the new system provides a measurement of $n \leq 4$ in band $R, n \leq 3$ in bands $Y, G$, and P. High field side (HFS) poloidal structural coverage is provided by bands $\mathrm{B}$ and allows resolution of modes with poloidal wavelengths as small as $14.2 \mathrm{~cm}$. Furthermore, in all locations both the radial and poloidal response are measured.

For understanding applied 3D field perturbations DIII-D is also equipped with two types of picture frame active coils. The first is a set of six coils outside of the vacuum vessel at the outer midplane of the machine. These coils are called the $\mathrm{C}$-coils and are predominantly used for correcting known error fields. The location of the C-coils is illustrated in Fig. 1. The second coil type is located inside the vacuum vessel, but behind graphite tile armor, and is referred to as the internal coils or I-coils. There are two arrays of I-coils, each array contains six coils distributed evenly in the toroidal direction. These coils are located above and below the outer midplane (Regions G in Fig. 1). Both the I- and C-coils are capable of generating toroidal perturbations with $\mathrm{n} \leq 3$. Also, a variety of poloidal spectra are possible by varying the toroidal phase of the applied perturbation between the coil rows. The amplitudes of applied and intrinsic 3D fields are expected to range between $<0.1 \mathrm{G}$, for edge localized $\mathrm{n}=3 \mathrm{RMP}$ perturbations, to hundreds of $\mathrm{G}$, for locked mode growth. The majority of the upgrade measures relatively slow plasma evolution with timescales greater than the vacuum vessel wall time of $3 \mathrm{~ms}$. For this reason, many of the sensors have effective bandwidths less than $20 \mathrm{kHz}$. To study higher poloidal wavenumber $(k)$ physics, the size of the sensors is kept small. Both the radial and poloidal field components are measured, allowing direct measurement of the field structure at each location, as well as providing an estimate of torques on the wall due to Maxwell stress.

This article is structured as follows: Sec. II shows modeling results, which provide a basis for determining the sensor sizes and placement needed to capture all physics goals. Section III provides an overview of the new sensor designs and how they are arranged. Section IV gives a detailed description of how the sensors were fabricated and installed with the necessary tolerances. Section V outlines an analog electronic signal-processing card designed for small 3D field recovery. Section VI describes an analysis method used for determining the plasma response from actively probed perturbations. Section VII shows a sample locked mode measurement using the new diagnostic upgrade, which agrees with existing sensor measurements.

\section{3D MODELING BASIS}

3D model predictions provide a basis for determining sensor locations and dimensions. The plasma response resulting from $n=1$ and $n=3$ RMPs where calculated using perturbed (MARS-F $\mathrm{F}^{17}$ and IPEC $^{18}$ ) as well as 3D equilibrium $\left(\mathrm{VMEC}^{19}\right)$ codes. The most detailed eigenstructure was found to be that of the $n=3$ RMP predicted by the linear ideal MHD code MARS-F. The smallest response amplitude was predicted by VMEC. To ensure the upgrade was capable of resolving either of these extremes the design criteria were set by the high spatial mode structure predicted by MARS-F and with the small perturbation amplitude predicted by VMEC.

MARS-F shows significant fine mode structure on the HFS, which is not predicted on the LFS of the machine. An example MARS-F prediction of the fine plasma response structure for an RMP ELM suppression $n=3$ I-coil perturbation is shown in Fig. 2(a). This motivated locating the majority of the new sensors on the HFS, since previous nonaxisymmetric magnetic measurements were entirely located on the LFS.

Figure 2(b) shows the MARS-F computed plasma response amplitude spectra for the $\mathrm{n}=3$ RMP ELM suppression along the vacuum vessel inner wall. These spectra are obtained by taking the spatial Fourier transform of the predicted response at a single toroidal angle. Note, the spectra are plotted with respect to the poloidal wavenumber $(k)$, which was taken to be the poloidal angle subtended along the inner vacuum vessel wall and has units of radians per meter. A range of poloidal spectral changes can be seen. Plasma pressure $\left(\beta_{\mathrm{N}}\right)$, edge safety factor $\left(\mathrm{q}_{95}\right)$, and the relative phase difference between upper and lower I-coil currents (parity) are varied. Here the plasma pressure is described by normalized beta $\beta_{N} \equiv \beta /(I / a B)$, where $\beta$ is the kinetic plasma pressure over the magnetic pressure, $I$ is plasma current, $a$ is minor radius, and $B$ is toroidal field. These ideal MHD predictions have 

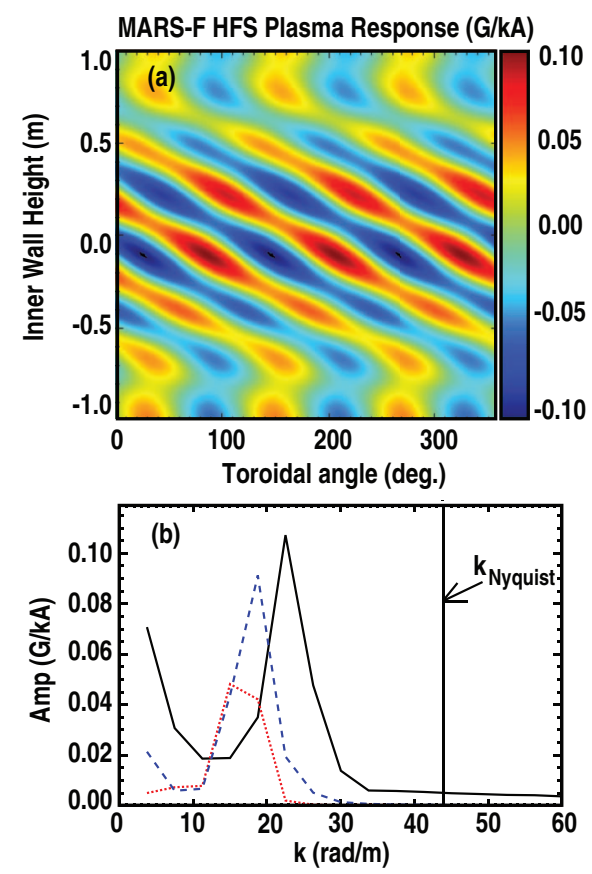

FIG. 2. $n=3$ MARS-F predicted RMP driven by I-coils, showing (a) plasma response along the DIII-D vacuum vessel inner wall for even parity, $\mathrm{q}_{95}$ $=3.5, \beta_{\mathrm{N}}=2.9$ and (b) poloidal spectra for: odd parity, $\mathrm{q}_{95}=5.0$, $\beta_{\mathrm{N}}=1.7$ (solid); even parity, $\mathrm{q}_{95}=3.5, \beta_{\mathrm{N}}=1.2$ (dotted); even parity, $\mathrm{q}_{95}=3.5, \beta_{\mathrm{N}}=2.9$ (dashed).

zero plasma rotation and resistivity. The initial EFIT equilibria inputs to MARS-F were constrained by magnetics, ${ }^{20}$ motional Stark effect ${ }^{21}$ (internal field pitch), charge exchange recombination ${ }^{22,23}$ (ion rotation and temperature), and Thomson scattering ${ }^{24}$ (electron temperature and density) diagnostics. The peak amplitude at $\mathrm{k}=18 \mathrm{~m}^{-1}$ doubles as the plasma pressure is scaled from $\beta_{\mathrm{N}}=1.2$ to $\beta_{\mathrm{N}}=2.9$. This is consistent with previous measurement and modeling of the outer wall plasma response. ${ }^{9,25}$ For larger $\mathrm{q}_{95}$ the dominant peak shifts to $\mathrm{k}=23 \mathrm{~m}^{-1}$, and a comparable response appears at $k=3 \mathrm{~m}^{-1}$. The sensor spacing is $\delta_{\mathrm{Z}}=14.2 \mathrm{~cm}$ for the vertical array. By definition, the Nyquist wavenumber $k_{\text {Nyquist }}$ $=\pi / \delta_{\mathrm{Z}}$. However, for reasons discussed in Sec. VI, the resulting Nyquist wavenumber for two component, co-located field measurements provides twice the spatial resolution with $k_{\text {Nyquist }}=2 \pi / \delta_{\mathrm{Z}}=44 \mathrm{~m}^{-1}$. This close effective spacing allows all dominant structural components for all these parameter variations to be captured without spatial aliasing. These short wavelength perturbations are predicted to be $<0.1 \mathrm{G}$, which is many orders of magnitude smaller than equilibrium field $\left(10^{-5}<\delta B / B_{0}<10^{-4}\right)$. Recovering these subGauss signals requires additional instrumentation discussed in Sec. V.

\section{SENSOR DESIGN AND LAYOUT}

Each sensor measurement $(\delta B)$ can be described as a linear combination of toroidal $(n)$ and poloidal $(m)$ harmonics,

$$
\delta B(\theta, \phi)=\sum_{m, n} B_{m, n} e^{i(m \theta+n \phi)} .
$$

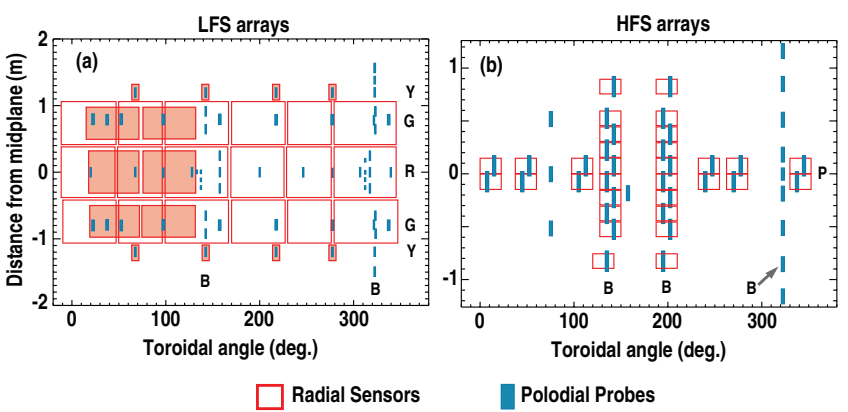

FIG. 3. Vacuum vessel wall of DIII-D showing internal magnetic diagnostics relevant to non-axisymmetric measurement: radial field sensors (open rectangles), poloidal field probes (solid rectangles). The vessel wall is presented as "unwrapped" with the vertical axis representing the poloidal distance from the midplane for both the (a) LFS and (b) HFS. Array band coding Y, G, R, $\mathrm{B}$, and $\mathrm{P}$ from Fig. 1 is repeated.

Here $B_{m, n}$ is complex, allowing the eigenmode amplitude and phase to be recovered, $\theta$ is the poloidal and $\phi$ is the toroidal location of a sensor, and $e^{i(m \theta+n \phi)}$ identifies the sinusoidal basis functions. For all non-rotating 3D eigenstructures consisting of $N \times M$ mode components, $2(N+1)(M+1)-1$ sensors provide a closed system of equations. Assuming only a single poloidal mode is present, then a toroidal sensor array consisting of $2 N+1$ sensors can provide a simultaneous measurement of $N$ toroidal mode components. Similarly, for 1 toroidal mode $2 M+1$ sensors resolves up to $M$ poloidal modes.

A sensor location map is shown in Fig. 3. Saddle loop radial field sensors (open rectangles) in Fig. 3(a), between $-1 \mathrm{~m}$ and $1 \mathrm{~m}$, provide three toroidal arrays of eight sensors. The shaded saddle loops at each poloidal location near $70^{\circ}$ toroidal angle overlap the other loops. Poloidal probe sensors (solid rectangles) provide simultaneous toroidal resolution up to $\mathrm{n}=3$. The 10 probes on the LFS midplane allow resolution up to $n=4$. The four-sensor toroidal arrays above $1 \mathrm{~m}$ and below $-1 \mathrm{~m}$ provide simultaneous measurement of $\mathrm{n}=0$ and 1 ; however, using synchronous detection, single mode numbers up to at least $\mathrm{n}=4$ may be resolved. Note, each of these locations contains both a poloidal probe and radial field sensor.

An irregular spacing was adopted for the toroidal sensor arrays to avoid degeneracies and allow detection of a wider range of mode numbers. An example of this spacing can be seen in array P of Fig. 3(b). Within the constraints of available space and compatibility with the vertical arrays [B arrays of Fig. 3(b)], the sensor locations were optimized for the desired combinations of toroidal mode numbers. The figure of merit is the condition number of the coupling matrix between the sensors and a given set of toroidal harmonics. (A smaller condition number is better.) With the as-built locations, the HFS toroidal arrays have a condition number of 2.38 for simultaneous resolution of $\mathrm{n}=(0,1,2,3)$, and 1.94 for simultaneously resolution of $n=(0,2,4)$. The latter combination is of interest for $\mathrm{n}=2$ plasma response measurements, since DIII-D's non-axisymmetric coil sets have a significant $\mathrm{n}=4$ harmonic when configured to apply an $n=2$ field. The arrays only contain eight sensors; however, irregular spacing extends the capability beyond the $2 \mathrm{~N}+1$ criterion for $\mathrm{n}=4$ resolution only 
TABLE I. Summary of DIII-D non-axisymmetric magnetic diagnostic sensors.

\begin{tabular}{|c|c|c|c|c|c|}
\hline Sensor type & Poloidal location (s) & No. of sensors & $\mathrm{NA}\left(\mathrm{cm}^{2}\right)^{\mathrm{a}}$ & $\mathrm{RC} / \mathrm{G}(\mathrm{ms})$ & $\mathrm{SNR}^{\mathrm{b}}$ \\
\hline$B_{p}$ probe: toroidal array & LFS $0 \mathrm{~m}$ & 10 & $1000-1200$ & 0.5 & 20 \\
\hline Saddle loop: toroidal array & LFS $0 \mathrm{~m}$ & 8 & $\sim 35000$ & 10.0 & 29 \\
\hline$B_{p}$ probe: toroidal arrays & $\mathrm{LFS} \pm 0.75 \mathrm{~m}$ & 16 & $2600-2700$ & 1.0 & 23 \\
\hline Saddle loop: toroidal arrays & $\mathrm{LFS} \pm 0.75 \mathrm{~m}$ & 16 & $26000-48000$ & 10.0 & 30 \\
\hline$B_{p}$ probe: toroidal arrays & $\mathrm{LFS} \pm 1.2 \mathrm{~m}$ & 8 & $4200-4240$ & 1.0 & 36 \\
\hline$B_{R}$ probe: toroidal arrays & $\mathrm{LFS} \pm 1.2 \mathrm{~m}$ & 8 & $11060-11420$ & 5.0 & 19 \\
\hline$B_{p}$ probe: toroidal and vertical arrays & HFS & 32 & 1950-1960 & 1.0 & 17 \\
\hline Saddle loop: toroidal and vertical arrays & HFS & 32 & 4860 & 2.0 & 20 \\
\hline$B_{p}$ probe: poloidal arrays & HFS and LFS & 62 & $100-1240$ & 0.5 & 20 \\
\hline$\dot{B}_{p}$ probe: short wavelength & LFS midplane & 8 & $240-250$ & $\ldots$ & $\ldots$ \\
\hline
\end{tabular}

${ }^{a}$ The NA for paired sensors is the total for both sensors.

${ }^{\mathrm{b}}$ The signal to noise ratio considering a $0.04 \mathrm{G}$ minimum expected signal and nominal RMS electronic noise $3 \mathrm{mV}$, and averaging over $200 \mathrm{~ms}$ data window.

if odd harmonics are negligible. For comparison, equal spacing of the eight sensors would yield a slightly better condition number of 1.41 for simultaneous resolution of $n=(0,1,2,3)$, but the spatial degeneracy would lead to an infinite condition number for any combination that includes $n=4$. A similar optimization was used in designing the LFS toroidal arrays, with the additional constraint of including existing sensors.

The $14.2 \mathrm{~cm}$ HFS vertical spacing of the B arrays at toroidal angle $142^{\circ}$ and $202^{\circ}$ were chosen based on modeling constraints described in Sec. II. A $28 \mathrm{~cm}$ gap to the last sensors at the top and bottom was used to better constrain $\mathrm{n}$ $<3$ spectra. The $60^{\circ}$ toroidal separation of these arrays was chosen to optimize the $\mathrm{n}=3$ difference amplitude while also allowing single-mode measurements of $\mathrm{n}=1$ and 2. Differencing will be discussed in more detail in Sec. VI, but it is important to note that the plasma response is known to decrease with increasing toroidal mode number. ${ }^{25}$ For typical plasma shapes, model predictions showed the HFS signal is peaked near the midplane, so the toroidal arrays on the HFS were located just above and below midplane. Furthermore comparison of the two toroidal arrays allows a simultaneous local estimate of the poloidal wavelength for $1 \leq \mathrm{n} \leq 3$.

Axisymmetric equilibrium reconstructions at DIII-D currently use an array of poloidal field $\left(\mathrm{B}_{\mathrm{P}}\right)$ probes at toroidal angle $322^{\circ}$. These sensors cover all poloidal angles of the machine. An appreciable $\mathrm{n}=1$ error field has been well documented in DIII-D. ${ }^{26}$ To provide immunity of the $2 \mathrm{D}$ equilibrium reconstruction from this dominant $\mathrm{n}=1$ error field, sensors were added to complete a second poloidal array $\sim 180^{\circ}$ away from the original array. This can be seen on the LFS at $142^{\circ}$, while the HFS utilizes the vertical array placement at $142^{\circ}$.

Closely spaced, high bandwidth sensors were installed at $135^{\circ}$ and $315^{\circ}$ on the LFS midplane. These high-resolution arrays are used to measure small wavelength instabilities (ELM precursors, TAEs, etc.). They are spaced such that poloidal mode wavelengths with $m<120$ may be measured.

The active area for measuring a change in magnetic flux is the area (A) normal to the change in flux multiplied by the number of turns $(\mathrm{N})$. The cross-sectional area is limited by the wavelength of interest. For many of the sensors the dimensions are selected to be no larger than $25 \%$ the wavelength of interest, to avoid averaging out the response. Even with fixed A, the sensitivity of any magnetic measurement can be made arbitrarily large by increasing $\mathrm{N}$. The finite diameter of the cabling provides an upper limit on the space a sensor can practically occupy. A summary of all sensor effective areas is shown in Table I.

Toroidal position location errors were determined to be an insensitive parameter for global non-axisymmetric measurements. The toroidal position is measured to within $0.1^{\circ}$, while a Monte Carlo study showed less than $7 \%$ error in the plasma response for toroidal position uncertainties as large as $2^{\circ}$.

\section{FABRICATION AND INSTALLATION}

Like the existing sensors, all new sensors were constructed using high temperature coaxial cable. This cable is composed of a 0.006-0.010 in. thick 316 stainless steel (SST) sheath, $\mathrm{MgO}$ ceramic insulator, and 0.010 in. copper center conductor. The thicker SST sheath cabling is used for saddle loops and radial field sensors where bandwidth is limited by the wall time of DIII-D ( $\sim 3 \mathrm{~ms})$ and not the sensor itself. An outer diameter of 0.063 in. was chosen for durability during disruptions. ${ }^{20}$ Additional robustness is provided by running twisted lead pairs directly to brazed vacuum feed through couplings, which avoids the potential for internal disconnections.

Figure 4 shows photos of three sensor designs. Figure 4(a) shows a poloidal sensor wound on a 316 SST frame. The windings enclose the frame. The conducting frame material results in eddy currents that reduce the bandwidth of the sensor by a factor of two $\left(f_{3 d B} \sim 20 \mathrm{kHz}\right)$ from previous designs. ${ }^{20,27}$ Despite this loss in bandwidth, the enclosed frame allows better winding accuracy and more careful centering of the return conductor to avoid radial field pickup. The "race-track" windings enclose the frame to maximize the cross-section that will fit under the tile armor.

On the HFS, misalignment of saddle loop windings on the order of a single cable diameter can result in erroneous pickup of $\mathrm{n}=0$ field up to $\sim 30 \mathrm{G}$. To achieve the greatest fabrication precision, the cable was pressed into a permanent frame containing channels the width of a cable diameter. The entire assembly, shown in Fig. 4(b), was installed. A tight 


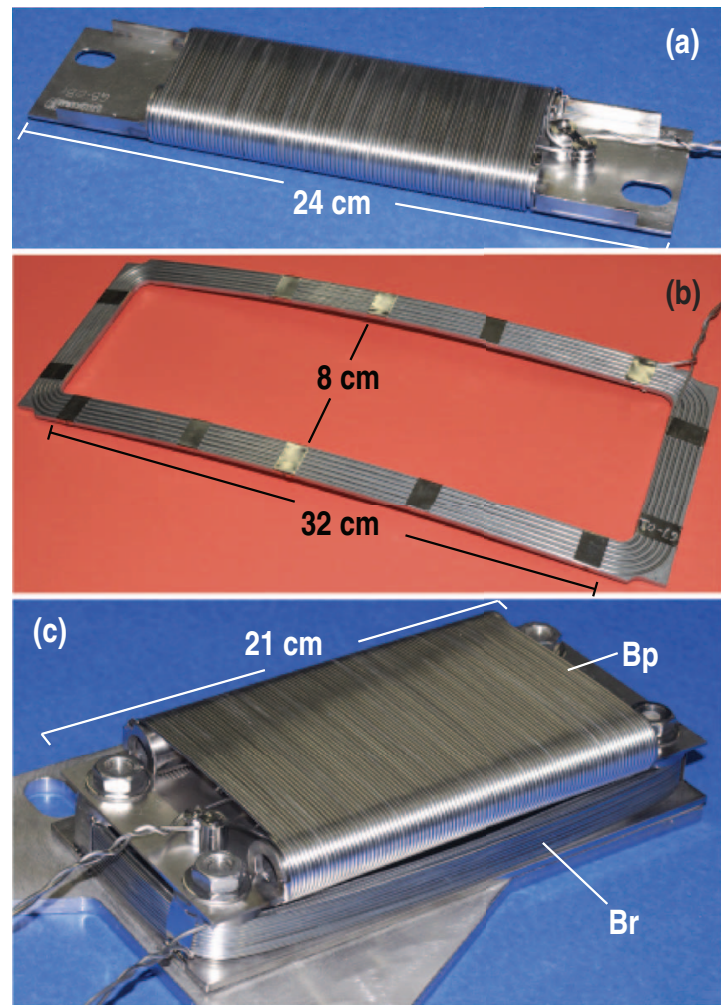

FIG. 4. (a) Poloidal sensor for HFS measurement. (b) HFS saddle loop and frame. This frame was precision-machined using electric discharge machining. (c) Poloidal and multi-turn radial field sensor.

tolerance, $10 \%$ the width of a cable diameter, was achieved by electric discharge machining (EDM) a flat piece of SST, then rolling the frame to the approximate curvature of the DIII-D inner wall. A 310 SST was selected for its reduced ferromagnetic properties during cold working.

Due to spatial constraints, a nested $\mathrm{Bp}-\mathrm{Br}$ design was employed above and below $\sim 1.2 \mathrm{~m}$ from the midplane on the LFS. This sensor assembly, pictured in Fig. 4(c), uses a radial field sensor comprised of multiple turns and layers of windings to boost NA in a compact space.

The sensors were aligned to gravity using a self-leveling rotatory laser level. The imaged shadows of the top windings of each senor were aligned vertically.
Sensor alignment and positions were confirmed using a coordinate measurement machine (CMM). Due to sparse landmark measurement points within the DIII-D vacuum vessel, and the need for high relative precision, a cylindrical fit was used to obtain an accurate $\mathrm{z}$-axis orientation and radial origin for the DIII-D coordinate system. The CMM arm was securely affixed at an arbitrary location within the DIII-D vacuum vessel. Hundreds of points were measured along the walls surface spanning the full extent of the CMM arm's reach. These data were fitted to a cylinder providing the $\mathrm{z}$-vector orientation. The absolute $\mathrm{z}$ was set to zero using a datum punch mark applied during vacuum vessel construction. The CMM arm is not large enough to measure all sensor positions from one location and was moved twice from its original position. At each new position a cylindrical fit was generated, and re-measurement of two sensors from the previous position maintained a constant coordinate system. Repeat measurements showed position uncertainty of $500 \mu \mathrm{m}$ and all sensors were vertically aligned to within this uncertainty. For HFS poloidal field sensors near the midplane this vertical alignment precision results in residual equilibrium $n$ $=0$ pickup less than $2 \mathrm{G}$.

\section{INSTRUMENTATION}

Finite digitizer bit resolution requires that most of the equilibrium and toroidal field detected by the sensors be removed. A D-tAcq ACQ196 16-bit digitizer with dynamic range $\pm 10 \mathrm{~V}$ has a bit resolution of $0.3 \mathrm{mV} / \mathrm{bit}$. Signal gains are set to span $25 \%$ of the full dynamic range for standard equilibrium field measurements. For $\delta B / B_{0} \sim 0.5 \times 10^{-4}$ the non-axisymmetric component of the field would span less than a digitizer bit.

The greatest 3D field resolution is provided through the use of signal-processing cards to remove the equilibrium poloidal and toroidal field. A schematic diagram of this 3D signal-processing card is shown in Fig. 5. Each sensor signal $(\phi)$ is detecting a combination of the axisymmetric equilibrium flux (either radial or poloidal and denoted by $\phi_{0}$ ), the 3D flux $\left(\phi_{3 D}\right)$ and some toroidal flux $\left(\phi_{B t}^{p}\right)$, resulting from imperfect alignment. A difference of the signals at the same

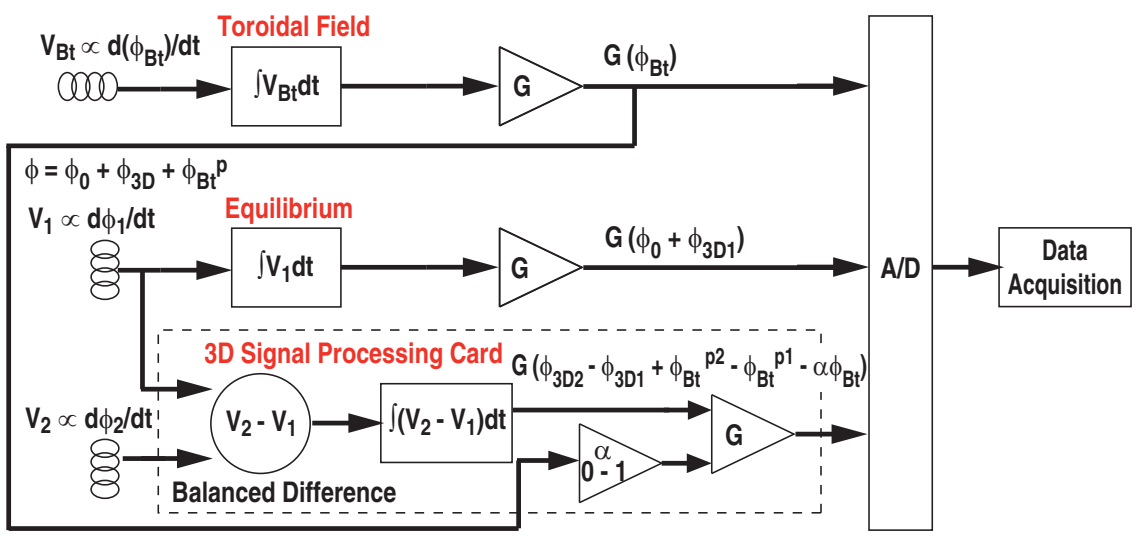

FIG. 5. Diagram of magnetic equilibrium and non-axisymmetric signal processing electronics. Toroidal field (top) is integrated. The equilibrium path (middle) integrates both equilibrium and non-axisymmetric components of the field before being amplified and digitized. 3D signal-processing card path (bottom) shows the unintegrated signals are differenced, then integrated, a toroidal field compensation signal is scaled then subtracted before amplification and digitization. 
poloidal location removes the majority of the axisymmetric equilibrium field. Subscripts and superscripts 1 and 2 in Figure 5 correspond to the first and second sensor in a difference pair, respectively. The relatively small misalignments mentioned in Sec. IV give rise to unique sensor-pair residual $\mathrm{n}=0$ pick-up after differencing. Also variations in NA of the sensors in the pair, due to imperfect fabrication, are not eliminated with a simple difference. For this reason, an adjustable balancing circuit is used to null the remaining equilibrium field pick-up. Each sensor-pair has a distinct balance factor requiring separate signal-processing cards. Through dedicated vacuum field pulses each sensor-pair is balanced to minimize this $\mathrm{n}=0$ residual field. This balanced and differenced signal is then integrated.

Another feature of the signal-processing card is a toroidal field compensation circuit. An appreciable toroidal field can be detected if sensors are slightly misaligned. For instance, a $0.1^{\circ}$ misalignment in the toroidal direction results in about $50 \mathrm{G}$ of toroidal field pickup. Therefore, a single separate integrated signal, originating from a sensor with magnetic axis oriented along the toroidal direction, provides a measurement of toroidal field variations for all of the 3D signal-processing cards. This toroidal signal is scaled on each card to match the toroidal field pickup of each differenced sensor-pair, such that $\phi_{B t}^{p 2}-\phi_{B t}^{p 1}-\alpha \phi_{B t}=0$, where $\phi_{B t}^{p 1}$ and $\phi_{B t}^{p 2}$ are the toroidal flux pick-up due to a misalignment of the first and second sensor in the pair, respectively, $\alpha$ is a positive or negative gain between 0 and 1 determined from vacuum pulses of the toroidal field coils, and $\phi_{B t}$ is the toroidal flux measured by the dedicated toroidal field magnetic sensor.

Through the use of this analog signal-processing card, the desired 3D field components can span the full dynamic range of the digitizer. For an equilibrium poloidal field of $0.7 \mathrm{~T}$, a small $\delta \mathrm{B} / \mathrm{B}_{0} \sim 0.5 \times 10^{-4}$ perturbation now typically spans $\sim 50$ digitizer bits.

The integrator time constants, gains, and sensor effective areas (NA) were chosen to provide an appreciable signalto-noise ratio (SNR) for even the smallest predicted perturbations. MARS-F spectra in Fig. 2 show non-peak plasma response amplitudes as small as $0.01 \mathrm{G} / \mathrm{kA}$. Electronic integrator circuits of similar architecture to those outlined in Fig. 5 showed random noise spanning $0.015 \%$ the dynamic range of the digitizer, or $3 \mathrm{mV}$. Averaging over the large timescale evolution (100-200 ms) of the 3D structure diminishes the effect of this noise by $\sqrt{N}$, where $N$ is the number of samples averaged. From Faraday's law the voltage digitized for any sensor is

$$
V_{d i g}=\delta B_{3 D} G\left(\frac{N A}{R C}\right),
$$

where $G$ is gain and $R C$ is the time constant of the integrator. The signal-to-noise ratio is described as $S N R$ $=V_{\text {dig }} \sqrt{N} / V_{\text {noise }}$. Table I summarizes the designed SNR assuming a $4 \mathrm{kA}$ applied field. Note, the impact of high frequency plasma fluctuations (e.g., sawteeth, rotating tearing modes, ELMs, etc.) on $\delta \mathrm{B}_{3 \mathrm{D}}$ measurement is not considered in this analysis.

Slow timescale integrator drift must be distinguished from actual 3D structural evolution. Repeatable, linear in- tegrator drift is removed by subtracting a previously determined slope and offset for the integrated signal of interest. Nonlinear, non-repeatable integrator drift is largely indistinguishable from the slow timescale evolution of the plasma. Many measurements of the drift made over a 10 s period, consistent with the length of a DIII-D shot interval, were used to determine a spectrum of possible drift waveforms. From this spectrum it was found that the drift waveforms roll off sharply after $4 \mathrm{~Hz}$. Probing the plasma with applied frequencies greater than the $4 \mathrm{~Hz}$ and recovering only the frequency of the perturbation avoids non-linear, non-repeating drift signal degradation. This synchronous detection technique is used when the signal of interest is comparable to the integrator drift amplitude.

\section{PLASMA RESPONSE ANALYSIS}

At present synchronous detection is needed to measure applied perturbation structure. This involves probing the plasma with an oscillating applied field, and determining the plasma response through Fourier analysis with respect to the applied reference frequency of the perturbing coil. Such measurements can be used for MHD spectroscopy, ${ }^{8}$ in which frequency-dependent response allows determination of the damping rate and natural frequency of stable plasma modes. Reducing integrator drift in a future upgrade of the instrumentation will allow static time evolution measurements of the 3D fields. Such an improvement will allow for full 3D equilibrium reconstructions.

Plasma response fitting is achieved through a number of steps including pre-processing and Fourier analysis. The raw data are first passed through a Kaiser digital low pass filter with corner frequency 50\% larger than the probing frequency. The order of the filter is sufficiently large to maintain the amplitude and phase of the probed signal to within $1 \%$ while rejecting low frequency integrator drift and high frequency electronic noise. The applied vacuum field pickup of the sensors must be subtracted from the total field measurement. The same filtering is applied to the perturbing coil current monitors prior to vacuum field compensation. Amplitude and phase values for vacuum coupling coefficients are determined through dedicated vacuum coil pulse shots at the perturbing frequency. After subtracting vacuum coupling, the plasma response is temporally Fourier analyzed over an integer number $(\geq 1)$ of periods of the perturbation to recover a single time-slice. These slices may be further spatially resolved to obtain the final eigenstructure.

Simultaneous solution of $M \times N$ spectral components may be obtained from Eq. (1) if each individual sensor measurement $(\delta B)$ were recorded. However, for practical reasons discussed in the previous section it is necessary to consider only sensor-pair differences to effectively recover small 3D magnetic response signals. For this reason, each pair difference is included explicitly in the resulting basis array, since $\Delta B=\delta B_{1}\left(\theta_{1}, \phi_{1}\right)-\delta B_{2}\left(\theta_{2}, \phi_{2}\right)$. The elements of the basis are limited to sine and cosine differences for each sensor location and mode combination. In other words, each sensorpair measurement $(\Delta B)$ can be described as a linear combination of toroidal $(n)$ and poloidal $(m)$ modes, $\Delta B$ 
$=\sum_{m, n} B_{m, n}\left(e^{i\left(m \theta_{1}+n \phi_{1}\right)}-e^{i\left(m \theta_{2}+n \phi_{2}\right)}\right)$. An example basis element for an $\mathrm{m}=2, \mathrm{n}=1$ mode corresponding to a difference pair of sensors located at $\left(\theta_{1}, \phi_{1}\right)$ and $\left(\theta_{2}, \phi_{2}\right)$ is $\cos \left(2 \theta_{2}\right.$ $\left.+\phi_{2}\right)-\cos \left(2 \theta_{1}+\phi_{1}\right)+i\left(\sin \left(2 \theta_{2}+\phi_{2}\right)-\sin \left(2 \theta_{1}+\right.\right.$ $\left.\phi_{1}\right)$ ). This provides the most general formulation; however, in DIII-D the differenced sensors are located at the same poloidal location such that $\theta_{1} \sim \theta_{2}$. The present analysis assumes the plasma boundary is circular. Future efforts are planned to map these elements to a realistic plasma 2D equilibrium. Also in this analysis, the finite dimension of the sensors is ignored, so each measurement is considered a point measurement of the local field.

The HFS arrays consist of co-located radial and vertical field measurements along the cylindrical inner wall. The sensors are located in a vacuum region between graphite tile armor and the vessel surface. Assuming field periodicity in $z$ and $\phi$ Lapace's equation collapses to the modified Bessel equation. Solving in this vacuum region gives a relationship between the measured radial and vertical field components,

$$
\mathrm{B}_{R}=i\left(\frac{I_{n}^{\prime}(k r)}{I_{n}(k r)}\right) B_{Z},
$$

where $I_{n}(k r)$ is the modified Bessel function of the first kind, $k$ is the poloidal wavenumber, and $r$ is major radius of the measurement. The general solution consists of a linear combination of Bessel functions of the first and second kind. In the vacuum region between the wall and the plasma at the high field side, the boundary conditions are such that the field from the plasma corresponds to the modified Bessel function of the first kind, and the field from induced currents in the wall to the second kind. Here we neglect the wall currents, assuming a slow time variation, leading to the simple form of Eq. (3). Equation (3) shows that the radial and vertical component of each toroidal mode is expected to have a $90^{\circ}$ phase difference. A poloidal mode can be described as a complex amplitude $B_{P M}$, where $B_{R}$ and $B_{Z}$ are the real and imaginary components, respectively, such that $B_{P M}=B_{R}+$ $i c(k r) B_{z}$ and $c(k r)$ is the Bessel function ratio from Eq. (3). Taking a discrete Fourier transform of this complex expression provides a spectrum with Nyquist wavenumber twice that of the spatial sampling interval for each single component measurement alone. In other words, measuring both components of the field at one location provides effective spatial resolution, without aliasing, equivalent to $14.2 \mathrm{~cm}$, despite the sensors being spaced $14.2 \mathrm{~cm}$ apart. The mode structure amplitude and phase can also be calculated directly at each location,

$$
\begin{gathered}
\phi=\tan ^{-1}\left(\sec n \varepsilon B_{z} / B_{R}-\tan n \varepsilon\right), \\
A=\gamma \sqrt{B_{Z}^{2}+B_{R}^{2}}, \\
\gamma=\sqrt{1+\frac{\sin 2 n \varepsilon}{2} \sin 2 \phi+\sin ^{2} n \varepsilon \cos 2 \phi,}
\end{gathered}
$$

where $\gamma$ is a geometric correction factor to compensate for a toroidal displacement $\varepsilon$ away from the co-located sensor position. It can be seen in Fig. 3(b) that the poloidal field sensors on the HFS have staggered toroidal positions such that $-3.25^{\circ}$ $<\varepsilon<3.25^{\circ}$ with respect to the radial field sensors center. Considering the HFS vertical array differenced signals, it is possible to use Eqs. (2)-(5) to recover the polodial spectrum of an applied perturbation assuming only a single toroidal mode exists.

\section{SAMPLE 3D MEASUREMENT}

Locking tearing modes provide a clear MHD structure, which can serve as a proxy to test the plasma response capabilities of the upgraded magnetics diagnostic without requiring careful vacuum compensation of direct pickup from active coils. A tearing mode-locking event is shown in Fig. 6(a). The $\mathrm{n}=1$ amplitude of the rotating mode increases as the rotation begins to decrease. Once the mode stops rotating the numerically integrated $d B / d t$ amplitude drops to zero. As the shielding effect of the wall is lost, slow timescale integrated radial field saddle loops show growth of the mode amplitude at roughly the vacuum vessel wall time. This locked mode grows until the plasma disrupts.

In Figs. 6(b) and 6(c), the structure of the non-rotating mode is compared with the structure observed earlier when the mode is rotating. The rotating mode structure is obtained through temporal Fourier analysis of unintegrated magnetic signals from an array of 14 poloidal sensors distributed

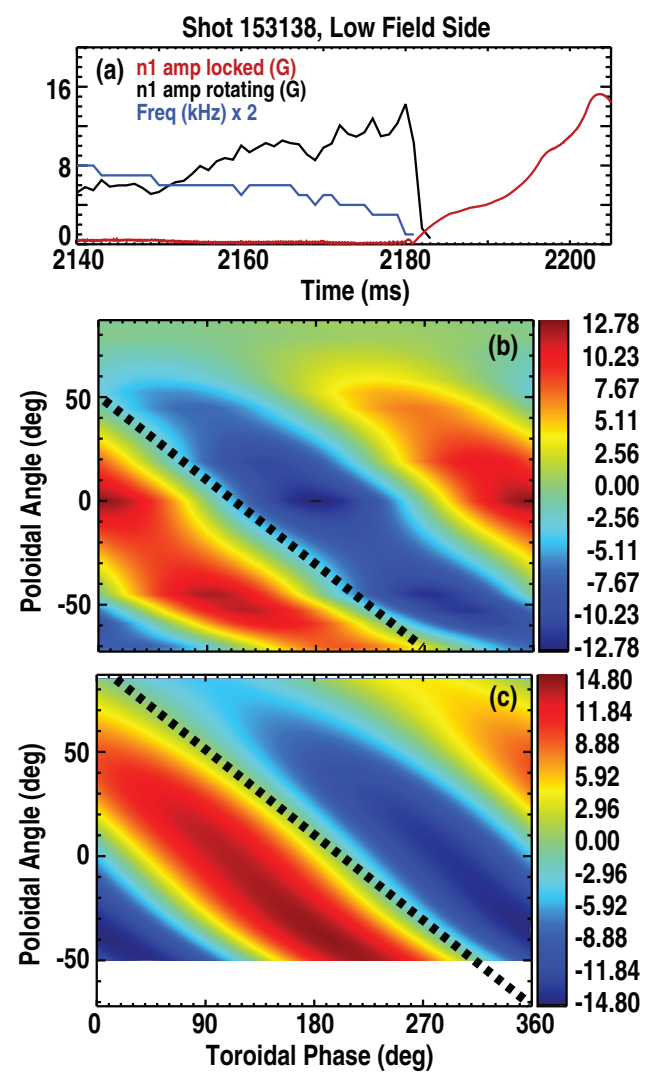

FIG. 6. (a) numerically integrated $n=1$ amplitude $\left(\delta \mathrm{B}_{\mathrm{p}}\right)$ of the rotating $\mathrm{n}=1$ tearing mode in Gauss, frequency of the rotating mode $\times 2 \mathrm{in} \mathrm{kHz}$, and a LFS saddle loop $\left(\delta \mathrm{B}_{\mathrm{r}}\right)$ measured $\mathrm{n}=1$ mode amplitude in Gauss appearing after the mode has locked. (b) Eigenstructure of a tearing mode rotating at $2.5 \mathrm{kHz}$ measured using unintegrated poloidal field probes on the LFS at $2170 \mathrm{~ms}$. Color bar in Gauss. Mode pitch (dashed) 2.2, consistent with a $\mathrm{m} / \mathrm{n}=2 / 1$. (c) Eigenstructure of the same tearing mode after locking to the wall, measured using integrated radial field sensors on the LFS at $2202 \mathrm{~ms}$. Color bar in Gauss. Mode pitch (dashed) 2.1, such that $\mathrm{m} / \mathrm{n}=2 / 1$. 
poloidally at $322^{\circ}$ toroidal angle. Figure $6(\mathrm{c})$ shows a sample measurement of the LFS structure of the non-rotating locked $2 / 1$ tearing mode prior to disruption, using 28 integrated radial field measurements distributed over the full poloidal and toroidal extent of the LFS vessel wall. The baseline field measured at $\mathrm{t}=2175 \mathrm{~ms}$ (before locking) is subtracted from the field measured at $2202 \mathrm{~ms}$ (after locking) to obtain a quasistatic $0<\mathrm{m}<6,0 \leq \mathrm{n} \leq 1$ mode fit. A clear pitch (toroidal angle/poloidal angle) of $\sim 2.1$ corresponds to a $2 / 1$ tearing mode. The overall structure is qualitatively the same between the two cases, with the rotating mode pitch estimated to be 2.2. Also, the locked mode peak amplitude quantitatively agrees with the rotating amplitude to within $14 \%$, following a couple wall times. The smaller amplitude in the rotating case, above $60^{\circ}$ poloidal angle, is a consequence of eddy current shielding caused by an upper baffle plate behind which the sensors are located. The non-rotating case is insensitive to the presence of the baffle because the mode dynamics are slower than the wall time.

Overall, this rotating vs. locked mode structural comparison provides great confidence that the 3D magnetic diagnostic upgrade accurately measures non-rotating non-axisymmetric fields. Furthermore, the upgrade is capable of measuring $\mathrm{n} \leq 3$ at seven poloidal locations (two locations on the HFS) and measuring poloidal wavelengths as small as 14.2 $\mathrm{cm}$. This will enable detailed 3D code validation and elucidate small 3D field physics. Experimental topics already exploiting this diagnostic are RMP ELM suppression, locked mode entrainment and avoidance, NRMF induced torque, error field identification and correction, and RWM control. It is expected that through the continued detail study of 3D fields, optimal non-axisymmetric perturbations will be found that extend the stable operating space of the tokamak.

\section{ACKNOWLEDGMENTS}

This material is based upon work supported by the U.S. Department of Energy, Office of Science, Office of Fusion Energy Sciences, using the DIII-D National Fusion Facility, a DOE Office of Science user facility, under Awards DE-FC0204ER54698, DE-AC05-06OR23100, DE-AC02-09CH11466, DE-AC02-09CH11466, and DE-AC05-00OR22725. DIII-D data shown in this paper can be obtained in digital format by following the links at https://fusion.gat.com/global/D3D. The authors are very grateful to the whole DIII-D team. The authors would like to give a special thanks to J. Kulchar and D. Ayala for their enormous fabrication efforts. Additional gratitude is also due to the operations staff of DIII-D, who went the extra mile to see this project succeed, namely: R. L. Lee, P. L. Taylor, A. G. Kellman, C. J. Murphy, B. Scoville, B. Brown, R. Tompkins, J. Burchett, E. Gonzales, D. Kellman, D. Piglowski, W. Carrig, and D. Whitaker. Also, thanks to D. Eldon for his graphics expertise. S. R. Haskey wishes to thank AINSE Ltd. for providing financial assistance.
${ }^{1}$ I. H. Hutchinson, Principles of Plasma Diagnostics (Cambridge University Press, Cambridge, 2002), Chap. 2.

${ }^{2}$ E. J. Strait, E. D. Fredrickson, J.-M. Moret, and M. Takechi, Fusion Sci. Technol. 53, 304 (2008).

${ }^{3}$ T. E. Evans, R. A. Moyer, P. R. Thomas, J. G. Watkins, T. H. Osborne, J. A. Boedo, E. J. Doyle, M. E. Fenstermacher, K. H. Finken, R. J. Groebner, M. Groth, J. H. Harris, R. J. La Haye, C. J. Lasnier, S. Masuzaki, N. Ohyabu, D. G. Pretty, T. L. Rhodes, H. Reimerdes, D. L. Rudakov, M. J. Schaffer, G. Wang, and L. Zeng, Phys. Rev. Lett. 92, 235003 (2004).

${ }^{4}$ A. M. Garofalo, K. H. Burrell, J. C. DeBoo, J. S. deGrassie, G. L. Jackson, M. J. Lanctot, H. Reimerdes, M. J. Shaffer, W. M. Solomon, and E. J. Strait, Phys. Rev. Lett. 101, 195005 (2008).

${ }^{5}$ A. M. Garofalo, W. M. Solomon, M. J. Lanctot, K. H. Burrell, J. C. DeBoo, J. S. deGrassie, G. L. Jackson, J.-K. Park, H. Reimerdes, M. J. Schaffer, and E. J. Strait, Phys. Plasmas 16, 056119 (2009).

${ }^{6}$ P. R. Brunsell, D. Yadikin, D. Gregoratto, R. Paccagnella, T. Bolzonella, M. Cavinato, M. Cecconello, J. R. Drake, A. Luchetta, G. Manduchi, G. Marchiori, L. Marrelli, P. Martin, A. Masiello, F. Milani, S. Ortolani, G. Spizzo, and P. Zanca, Phys. Rev. Lett. 93, 225001 (2004).

${ }^{7}$ R. Paccagnella, S. Ortolani, P. Zanca, A. Alfier, T. Bolzonella, L. Marrelli, M. E. Puiatti, G. Serianni, D. Terranova, M. Valisa, M. Agostini, L. Apolloni, F. Auriemma, F. Bonomo, A. Canton, L. Carraro, R. Cavazzana, M. Cavinato, P. Franz, E. Gazza, L. Grando, P. Innocente, R. Lorenzini, A. Luchetta, G. Manduchi, G. Marchiori, S. Martini, R. Pasqualotto, P. Piovesan, N. Pomaro, P. Scarin, G. Spizzo, M. Spolaore, C. Taliercio, N. Vianello, B. Zaniol, L. Zanotto, and M. Zuin, Phys. Rev. Lett. 97, 075001 (2006).

${ }^{8}$ H. Reimerdes, M. S. Chu, A. M. Garofalo, G. L. Jackson, R. J. La Haye, G. A. Navratil, M. Okabayashi, J. T. Scoville, and E. J. Strait, Phys. Rev. Lett. 93, 135002 (2004)

${ }^{9}$ M. J. Lanctot, H. Reimerdes, A. M. Garofalo, M. S. Chu, Y. Q. Liu, E. J. Strait, G. L. Jackson, R. J. La Haye, M. Okabayashi, T. H. Osborne, and M. J. Schaffer, Phys. Plasmas 17, 030701 (2010).

${ }^{10}$ M. F. F. Nave and J. A. Wesson, Nucl. Fusion 30, 2575 (1990).

${ }^{11}$ N. C. Logan, E. J. Strait, and H. Reimerdes, Plasma Phys. Controlled Fusion 52, 045013 (2010).

${ }^{12}$ L. L. Lao, J. R. Ferron, R. J. Groebner, W. Howl, H. St. John, E. J. Strait, and T. S. Taylor, Nucl. Fusion 30, 1035 (1990).

${ }^{13}$ K.-L. Wong, Plasma Phys. Controlled Fusion 41, R1 (1999).

${ }^{14}$ M. Okabayashi, J. Bialek, A. Bondeson, M. S. Chance, M. S. Chu, A. M. Garofalo, R. Hatcher, Y. In, G. L. Jackson, R. J. Jayakumar, T. H. Jensen, O. Katsuro-Hopkins, R. J. La Haye, Y. Q. Liu, G. A. Navratil, H. Reimerdes, J. T. Scoville, E. J. Strait, M. Takechi, A. D. Turnbull, P. Gohil, J. S. Kim, M. A. Makowski, J. Manickam and J. Menard, Nucl. Fusion 45, 1715 (2005).

${ }^{15}$ S. A. Sabbagh, R. E. Bell, J. E. Menard, D. A. Gates, A. C. Sontag, J. M. Bialek, B. P. LeBlanc, F. M. Levinton, K. Tritz, and H. Yuh, Phys. Rev. Lett. 97, 045004 (2006).

${ }^{16}$ M. S. Chu and M. Okabayashi, Plasma Phys. Controlled Fusion 52, 123001 (2010).

${ }^{17}$ Y. Q. Liu, A. Bondeson, C. M. Fransson, B. Lennartson, and C. Breitholtz, Phys. Plasmas 7, 3681 (2000).

${ }^{18}$ J.-K. Park, A. H. Boozer, and A. H. Glasser, Phys. Plasmas 14, 052110 (2007).

${ }^{19}$ S. Hirshman and J. Whitson, Phys. Fluids 26, 3553 (1983).

${ }^{20}$ E. J. Strait, Rev. Sci. Instrum. 77, 023502 (2006).

${ }^{21}$ F. M. Levinton, R. J. Fonck, G. M. Gammel, R. Kaita, H. W. Kugel, E. T. Powell, and D. W. Roberts, Phys. Rev. Lett. 63, 2060 (1989).

${ }^{22}$ R. C. Isler, Plasma Phys. Controlled Fusion 36, 171 (1994).

${ }^{23}$ R. P. Seraydarian and K. H. Burrell, Rev. Sci. Instrum. 57, 2012 (1986).

${ }^{24}$ P. K. Trost, T. N. Carlstrom, J. C. DeBoo, C. M. Greenfield, C. L. Hsieh, and R. T. Snider, Rev. Sci. Instrum. 61, 2864 (1990).

${ }^{25}$ M. J. Lanctot, H. Reimerdes, A. M. Garofalo, M. S. Chu, J. M. Hanson, Y. Q. Liu, G. A. Navratil, I. N. Bogatu, Y. In, G. L. Jackson, R. J. La Haye, M. Okabayashi, J.-K, Park, M. J. Schaffer, O. Schmitz, E. J. Strait, and A. D. Turnbull, Phys. Plasmas 18, 056121 (2011).

${ }^{26}$ J. L. Luxon, M. J. Schaffer, G. L. Jackson, J. A. Leuer, A. Nagy, J. T. Scoville, and E. J. Strait, Nucl. Fusion 43, 1813 (2003).

${ }^{27}$ A. S. Bozek and E. J. Strait, General Atomics Report GA-A24492, 2004 (unpublished); Proceedings of 29th IEEE/NPSS Symposium on Fusion Engineering, San Diego, California, 2003 (Institute of Electrical and Electronics Engineers, Inc., Piscataway, 2004), p. 336. 